\title{
Exchange Interactions in a Low-Dimensional Magnetic System $\mathrm{Cu}\left(\mathrm{H}_{2} \mathrm{O}\right)_{2}(\mathrm{en}) \mathrm{SO}_{4}$
}

\author{
R. SÝKORA ${ }^{a, *}$, D. LEGUT ${ }^{a}$, U.D. WDOWIK ${ }^{b}$ \\ ${ }^{a}$ Nanotechnology Centre, VSB-Technical University of Ostrava, 17. listopadu 15, 70833 Ostrava, Czech Republic \\ ${ }^{b}$ Institute of Technology, Pedagogical University, ul. Podchorazych 2, 30-084 Kraków, Poland
}

We report a theoretical ab-initio investigation of the exchange interaction between $\mathrm{Cu}$ atoms in $\mathrm{Cu}\left(\mathrm{H}_{2} \mathrm{O}\right)_{2}(\mathrm{en}) \mathrm{SO}_{4}$, en $=\mathrm{C}_{2} \mathrm{H}_{8} \mathrm{~N}_{2}$ (ethylenediamine), an insulating magnetic material. Unlike previous experimental studies which describe the system as a quasi-two-dimensional antiferromagnet, our results, based on the mapping of the system onto an effective Heisenberg model, suggest a quasi-one-dimensional character of magnetism, with the exchange coupling between the $\mathrm{Cu}$ atoms being propagated along a zigzag line, lying in the crystal's $b c$ plane and connecting the $\mathrm{Cu}$ atoms through the $\mathrm{N}$ atoms. Furthermore, we report the calculated positions of the $\mathrm{H}$ atoms that have not been provided in experimental papers.

DOI: $10.12693 /$ APhysPolA.126.50

PACS: 75.10.Hk, 75.10.-b, 75.40.Cx, 75.50.Ee

\section{Introduction}

Low-dimensional magnets with low critical temperatures feature a wealth of experimentally accessible quantum-mechanical phenomena, which are not blurred by temperature-related effects. Below we give a brief theoretical account of magnetic exchange coupling in $\mathrm{Cu}\left(\mathrm{H}_{2} \mathrm{O}\right)_{2}($ en $) \mathrm{SO}_{4}$, supposedly an insulating antiferromagnet with the Néel temperature of $T_{N}=0.9 \mathrm{~K}$ [1] Our results approve both the material being an insulator (calculations yield a gap of about $1 \mathrm{eV}$ ) and the antiferromagnetic order as a candidate for the ground state. Nonetheless our results also imply the system's effective magnetic coupling paths are different from those previously considered in [1] and form zigzag chains. This quasi-one-dimensional character makes the system appealing since explanation and quantification of phase transitions in such systems is not easy $[2,3]$.

\section{Computational methodology}

The calculations were performed using the VASP code $[4,5]$ within the density functional theory, using the projector-augmented-wave pseudopotential method with the generalized gradient approximation parameterized by Perdew, Burke, and Ernzerhof. The Brillouin zone was sampled on a $k$-point mesh with $5 \times 3 \times 4$ points (for supercells doubling the unit cell along the $a$, or $b$, or $a$ and $c$ directions, used when we considered more complicated antiferromagnetic orderings, this was $2 \times 2 \times 3$, $5 \times 1 \times 4$, and $3 \times 3 \times 2$, respectively). We used the conjugate gradient energy minimization scheme for geometry optimization and relaxed the structure until the forces on individual atoms were smaller than $5 \times 10^{-3} \mathrm{eV} \AA^{-1}$

*corresponding author; e-mail: rudolf .sykora@gmail.com and the external pressure was below 0.1 kbar. All the calculations presume collinear magnetism and ignore the spin-orbit coupling.

\section{Geometric structure}

The $\mathrm{Cu}\left(\mathrm{H}_{2} \mathrm{O}\right)_{2}($ en $) \mathrm{SO}_{4}$ possesses a monoclinic symmetry, space group $C 2 / c$ (no. 15), and four chemical formula units $(Z=4)$ in the unit cell, which thus contains 96 atoms [6]. The $\mathrm{Cu}$ and $\mathrm{S}$ atoms have the Wyckoff positions $4 \mathrm{e}$, all the others are at general $8 \mathrm{f}$ positions. The structure is formed by stacking, along the $c$ axis, of hydrogen-bonded $a b$ planes of mutually hydrogen-bonded linear chains running along the $a$ axis of covalently-bonded $\mathrm{Cu}\left(\mathrm{H}_{2} \mathrm{O}\right)_{2}($ en $) \mathrm{SO}_{4}$ complexes. Experimental lattice parameters together with our optimized ones are given in Tab. 1 . The calculated constants are larger by $+5,+2$, and $-1 \%$ in the $a, b, c$ dimensions, respectively.

\section{TABLE I}

Experimental [6] and optimized lattice parameters.

\begin{tabular}{c|c|c|c|c|c}
\hline \hline & $\mathrm{a}[\AA]$ & $\mathrm{b}[\AA]$ & $\mathrm{c}[\AA]$ & $\beta\left[^{\circ}\right]$ & $\mathrm{V}\left[\AA^{3}\right]$ \\
\hline exp. & 7.232 & 11.725 & 9.768 & 105.5 & 798 \\
\hline cal. & 7.597 & 11.964 & 9.675 & 104.0 & 853
\end{tabular}

Equilibrium atomic positions are given in Tab. 2, and are in a good agreement (to within $2 \%$ of the lattice vectors) with the X-ray diffraction values [6].

\section{Magnetic order}

For the optimized geometry we have evaluated the total energies of the system with ferromagnetically (FM) ordered $\mathrm{Cu}$ ions as well as for several antiferromagnetic (AF) configurations. The calculations show that magnetic moments exist only on the $\mathrm{Cu}$ atoms, of $0.6 \mu_{B}$ (Bohr magneton), and on $\mathrm{N}$ atoms, of $0.1 \mu_{B}$, the latter 
being always ferromagnetically ordered with respect to the closest $\mathrm{Cu}$ atom. These moments are stable between all the different investigated magnetic orderings. This allows us to map the system's energy differences onto an effective Heisenberg model, described by the Hamiltonian $H=-\frac{1}{2} \sum_{i \neq j} J_{i j} \boldsymbol{e}_{i} \cdot \boldsymbol{e}_{j}$, where $i, j$ count the $\mathrm{Cu}$ sites, $J_{i j}$ represent effective exchange couplings, and $\boldsymbol{e}_{i}$ denotes the site- $i$ magnetization direction, $\boldsymbol{e}_{i} \boldsymbol{e}_{j}= \pm 1$ for a FM and AF ordering, respectively. Hereafter, instead of $J_{i j} / 2$ we use $J n$ to label different exchange couplings.

TABLE II

Calculated equilibrium atomic positions given as fractional coordinates $x, y, z$ with respect to the (relaxed) $a, b, c$ lattice vectors. Only inequivalent positions are listed. In parentheses the experimental values [6] are given (these are, strictly speaking, with respect to the experimental lattice parameters).

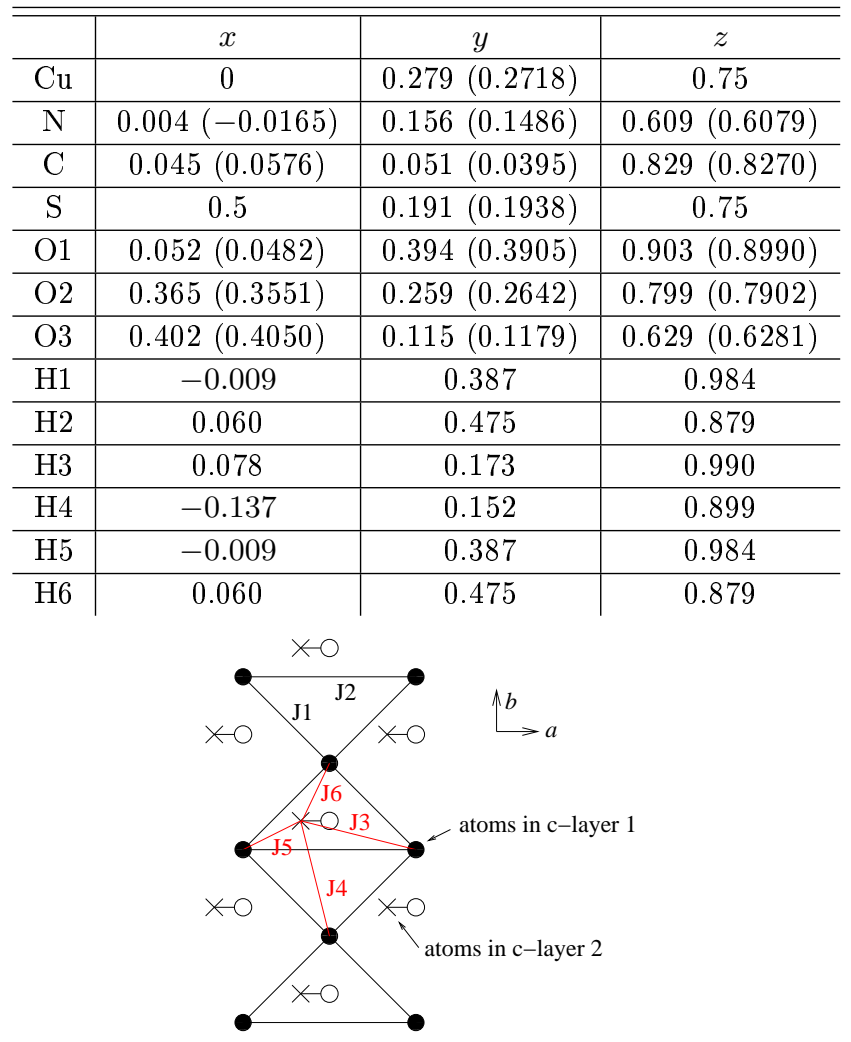

Fig. 1. Considered neighbours and exchange couplings of a $\mathrm{Cu}$ atom. Full circles represent atoms in one $c$ layer, crosses then atoms in the next $c$ layer, whose positions would be empty circles if $\beta$ were $90^{\circ}$. The distances corresponding to $J 1-J 6$ are $7.1,7.6,6.9,8.2,5.4,7.2 \AA$, respectively.

For each $\mathrm{Cu}$ atom, guided by the geometric distances, we considered its $14 \mathrm{Cu}$ neighbours, see Fig. 1. There are 6 neighbours in the same $c$ plane: 2 along the $a$ direction, within the same covalently-bonded chain, described by the exchange $J 2$, and $4(2+2)$ in the two neighbouring chains, described by $J 1$. Each $\mathrm{Cu}$ atom further has 8 neighbours in the $c$ planes above and below itself $(4+4)$, described by constants $J 3$ to $J 6$.
All of the considered exchange couplings with the exception of $J 3$ are antiferromagnetic, but most importantly only the $J 4$ constant is sizeable, of the order $-5 \times 10^{-4} \mathrm{eV}$, whereas the others being at least an order of magnitude smaller. Namely, $J 1 \approx-8 \times 10^{-6} \mathrm{eV}, J 2 \approx$ $-7 \times 10^{-7} \mathrm{eV}, J 3 \approx+6 \times 10^{-6} \mathrm{eV}, J 5 \approx-6 \times 10^{-6} \mathrm{eV}$, and $J 6 \approx-7 \times 10^{-5} \mathrm{eV} . J 4$ corresponds to the coupling via the $\mathrm{N}$ atoms, spans the longest distance, and forms a zigzag chain in the $b c$ plane.

\section{Conclusions}

Using first-principle calculations we determined exchange couplings in a low-dimensional antiferromagnetic insulator $\mathrm{Cu}\left(\mathrm{H}_{2} \mathrm{O}\right)_{2}($ en $) \mathrm{SO}_{4}$. The magnetic ordering is rather one-dimensional (along a zigzag chain within the $b c$ plane) than two-dimensional (in the $a b$ plane) as supposed previously [1]. Further, the calculation shows the gap of about $1 \mathrm{eV}$ indicating the true one can be expected at about $2 \mathrm{eV}$, in accord with a preliminary $\mathrm{UV}$ VIS-spectroscopy measurement (measured with Cintra 303 spectrometer at VSB-TUO Ostrava, Czech Republic). Finally, the positions of the $\mathrm{H}$ atoms that have not been reported before are given.

\section{Acknowledgments}

The access to the CERIT-SC computing facilities provided under the programme Center CERIT Scientific Cloud, reg. no. CZ. 1.05/3.2.00/08.0144, is appreciated. This research used resources of the National Supercomputing Center IT4Innovations, supported by the Op VaVpI project number CZ.1.05/1.1.00/02.0070. The paper has been elaborated in the framework of the project New creative teams in priorities of scientific research, reg. no. CZ.1.07/2.3.00/30.0055, supported by Operational Programme Education for Competitiveness and co-financed by the European Social Fund and the state budget of the Czech Republic. D. Legut was supported by the Grant Agency of the Czech Republic under reg. no. $13-30397 \mathrm{~S}$.

\section{References}

[1] M. Kajňaková, M. Orendáč, A. Orendáčová, A. Vlček, J. Černák, O.V. Kravchyna, A.G. Anders, M. Bałanda, J.-H. Park, A. Feher, M.W. Meisel, Phys. Rev. B 71, 014435 (2005).

[2] C. Yasuda, S. Todo, K. Hukushima, F. Alet, M. Keller, M. Troyer, H. Takayama, Phys. Rev. Lett. 94, 217201 (2005).

[3] B. Lake, D.A. Tennant, C.D. Frost, S.E. Nagler, Nature Materials 4, 329 (2005).

[4] G. Kresse, J. Furhmüller, Phys. Rev. B 54, 11169 (1996).

[5] G. Kresse, J. Furhmüller, Comput. Mater. Sci. 6, 15 (1996).

[6] V. Manríquez, M. Campos-Vallette, N. Lara, N. González-Tejeda, O. Wittke, G. Díaz, S. Diez, R. Munoz, L. Kriskovic, J. Chem. Crystall. 26, 15 (1996). 